\title{
Perceived Innovativeness and Privacy Risk of Smart Toys in Brazil and Argentina
}

\author{
Marcelo Fantinato ${ }^{1}$, Patrick C. K. Hung ${ }^{2}$, Ying Jiang ${ }^{2}$, Jorge Roa $^{3}$ \\ Pablo Villarreal ${ }^{3,4}$, Mohammed Melaisi ${ }^{2}$, Fernanda Amancio ${ }^{1}$ \\ ${ }^{1}$ School of Arts, Sciences and Humanities, University of São Paulo, Brazil \\ ${ }^{2}$ Faculty of Business and IT, University of Ontario Institute of Technology, Canada \\ ${ }^{3}$ CIDISI, UTN-FRSF, Argentina \\ ${ }^{4}$ CONICET, Argentina \\ Email: \{m.fantinato, f.amancio $\} @$ usp.br; \{jroa, pvillarr $\} @$ frsf.utn.edu.ar \\ \{patrick.hung, ying.jiang, mohammed.melaisi\} @uoit.ca
}

\begin{abstract}
A smart toy, such as "Hello Barbie," is a device consisting of a physical toy component that connects to a computing system with online services through networking to enhance the functionality of a traditional toy. Whilst these are new educational and entertaining values of smart toys, experts in western countries such as U.S. and Germany have warned consumers of the data security and privacy issues of these toys. In this preliminary research study, we particularly studied Brazilian and Argentinian consumers' perceived innovativeness, risks and benefits of smart toys and their purchase intention toward such toys. Results indicate that Brazilian consumers have better perception and evaluation of the toy and thus higher purchase intention than Argentinian consumers do. Such difference may be explained by the cultural differences between the two countries, such as relatively low vs. high uncertainty avoidance.
\end{abstract}

\section{Introduction}

After Amazon's Echo line of smart speakers powered by its Alexa virtual assistant system became the best-selling products on Amazon during the last holiday season [21], soon children will be able to have their own version of Echo, Smarty, a voice-controlled digital assistant designed particularly for kids [20]. Smarty is just one example of the many Internet-connected smart toys that appear in the market in recent years. Others include Mattel's Hello Barbie, CogniToys' Talking Dino, and Fisher-Price's Smart Toy Bear. UK-based Juniper Research has reported that smart toys are the new key market for toy companies and the sales of smart toys would grow from $\$ 2.8$ billion in 2015 to $\$ 11.3$ billion by 2020 [23].

These Internet-connected smart toys usually have a component that connects to a computing system with online services to enable voice recording, recognition, and database search. Therefore, a traditional teddy bear can now listen and talk back to a child intellectually. Whilst these are new educational and entertaining values of smart toys, experts have warned consumers of the data security and privacy issues of these toys. A recent U.S. Senate report states that these toys may gather a child's personal information, which may potentially cause serious consequences such as identity theft [25]. Likewise, the Federal Network Agency (Bundesnetzagentur) in Germany is telling parents to abandon Internet-connected smart toys designed for their kids because of it's insecure and hackable structure that could reveal personal information [3].

Prior research on data privacy shows that greater concern often leads to negative responses [26] and consumers often weigh the consequences of personal information disclosure against the value offered by the marketer [22]. However, most such research has primarily focused on western cultures and not much research has studied data privacy issues among Brazil, Russia, India and China (BRIC) countries [24]. Further, although smart toys have been getting their popularity in developed countries, they have not been widely introduced in emerging markets. The objectives of this research are to investigate: (1) whether consumers in emerging market such as Brazil and Argentina perceive the innovativeness, risks and benefits of the conversational function of smart toys differently, and (2) how such perceptions influence their overall evaluation of and purchase intention toward smart toys. Therefore, this research contributes to the literature of consumer data privacy by demonstrating the outcomes of data privacy concerns in 
Brazil and Argentina. Further, our research also adds to the literature of consumer new product adoption by demonstrating how perceived innovativeness of a product may have either positive or negative impact on product evaluation and purchase intention in different cultures.

This paper is organized as follows: Section 2 provides background information, Section 3 describes the research framework applied, Section 4 presents the results of our empirical study in Brazil and Argentina, and Section 5 concludes the paper with future work.

\section{Background Information}

Why are smart toys under scrutiny for data privacy and security? We illustrate this issue using the example of Hello Barbie. Hello Barbie is a smart toy manufactured by Mattel [7]. While the doll is made by Mattel Inc., the online conversation software is powered by ToyTalk. ToyTalk has previously released a smartphone application known as SpeakALegend, which allowed children to interact and engage in conversation with imaginary characters such as the unicorn, mermaid, and Bigfoot [7]. With their expertise in this field, Mattel cooperated with them to develop the software behind an interactive Hello Barbie. Referring to the vocabulary of Hello Barbie as of November 17, 2015, she can speak 56,367 total words and 3,935 unique word forms in 8,000 phrases.

Referring to Figure 1, the children interact with Hello Barbie equipped with WIFI, microphone, and speaker in a physical and social environment. When Hello Barbie turns on, the system inside the doll checks if the doll has been linked to a ToyTalk.com account via WIFI. For the parental control, the parents/guardians must download a mobile application called "Hello Barbie Companion App" on a smartphone to configure the
WIFI settings. The ToyTalk.com account provides the parents/guardians functions to manage the conversation options. Following that, the app asks the parents/guardians for their consent to allow the company to use their child's information, such as voices, their birthday and holidays they care about. If the parents refuse to give permission, ToyTalk.com will not store any information in the Cloud and the account will be deleted in a reasonable time. If the parents give permission, ToyTalk.com will have the right under their privacy policy to gather information from Hello Barbie or even other smart toys of the same account.

The conversation options allow parents to provide the doll with information of the child that is using the doll. The information consists of important holidays, such as Halloween, Thanksgiving, Christmas, Diwali and Hanukkah. Parents can also provide the child's day and month of birth to Hello Barbie. These options do not require the doll to be in connection mode, which means that the data is stored in the doll internally.

The physical interface between a child and a smart toy is usually via a touch, e.g., digital button [9]. After this point, the child should be able to engage in conversation with Hello Barbie via a button, while the parents can access the conversation audio clips via the ToyTalk.com account. Hello Barbie sends the collected voice in audio clips to ToyTalk.com services, and ToyTalk.com can bind with other third-party social networking services such as Facebook, Twitter, Pinterest, Tumblr and Instagram, in the Cloud. Both Mattel and ToyTalk.com have its own privacy policy that outlines information including how they collect, manage, share and retain the user's personal data.

Referring to Figure 2, ToyTalk.com services on the Cloud has a list of phrases that Hello Barbie is the one who is asking a question and waiting for a response. After that, Hello Barbie requests a phrase from ToyTalk's

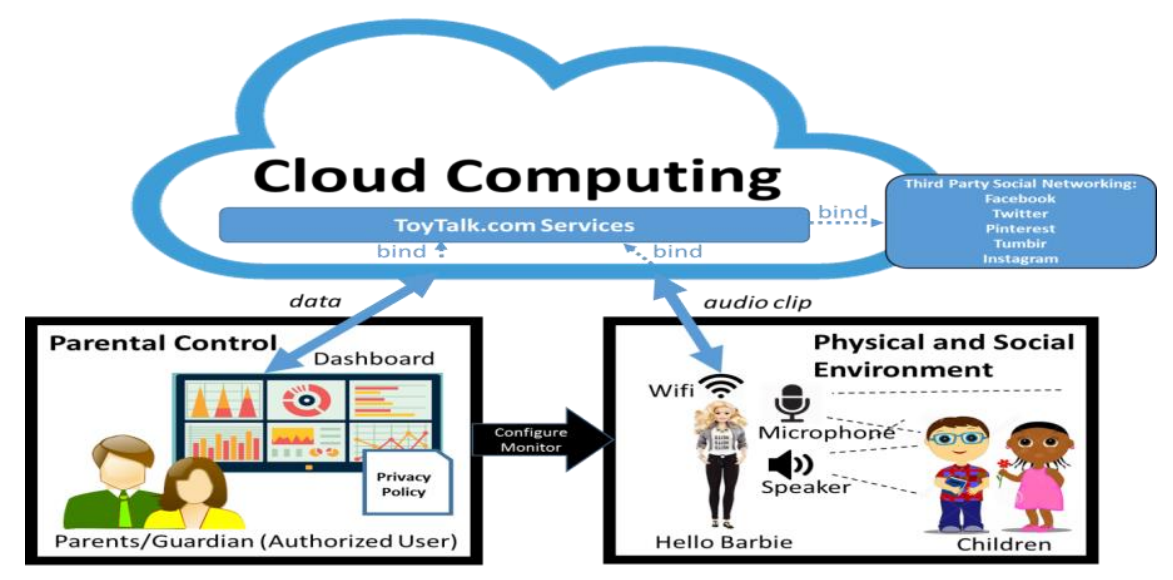

Figure 1. Conceptual Model of Hello Barbie and ToyTalk.com 


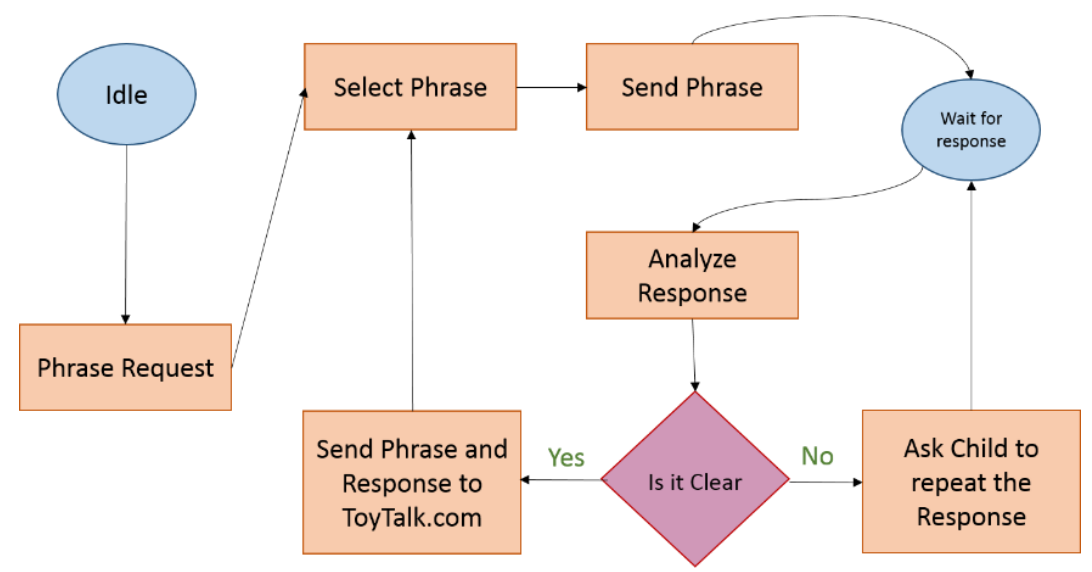

Figure 2. Hello Barbie Phrase Conversation

services and plays an audio response for the user. The conversations vary from talking about specific topics such as fashion, school, friends, and family, to playing games and listening to interactive stories. In addition, Hello Barbie tries to ask the user questions regarding these topics to engage them in the conversation. For example, the phrase "Well, we've been talking so much about school... what about all the things we can do when we're not in class? Let's talk about that!" intends to change the topic from talking about school to talk about hobbies or other interests. In this scenario, one can see that Hello Barbie may actively drive the flow of the conversation.

Referring to Figure 3, the speech recognition services on ToyTalk.com receives the child's recording and analyzes it to find the best response. Many conditions control the flow of the conversation. In the beginning, ToyTalk.com checks if the user has said phrases or words from a priority list. This list contains command phrases, such as volume up and down, which makes
Hello Barbie repeat the last statement in a lower or higher voice. Other phrases include Hello Barbie questions such as "Can I ask you a question?" Another type of conversation is a narrative interactive story. In this scenario, Hello Barbie gives the child two options to choose. If the child's answer is vague, Hello Barbie will ask the child again. If the child does not answer clearly for the second time, Hello Barbie will assume one option and carry on with her own story. In a regular topic conversation, Hello Barbie says something and then asks the child-related questions, such as what food they like, how they dressed for an event, what they like about school etc. In this type of conversation, Hello Barbie asks general questions and does not change her behavior based on the answer. Hello Barbie will remember a few things, such as whether the child has a pet. Another thing Hello Barbie can remember is the last conversation or a previous game played with the user, in which case Hello Barbie says something like "Do you remember when we did this?" This kind of memory might help

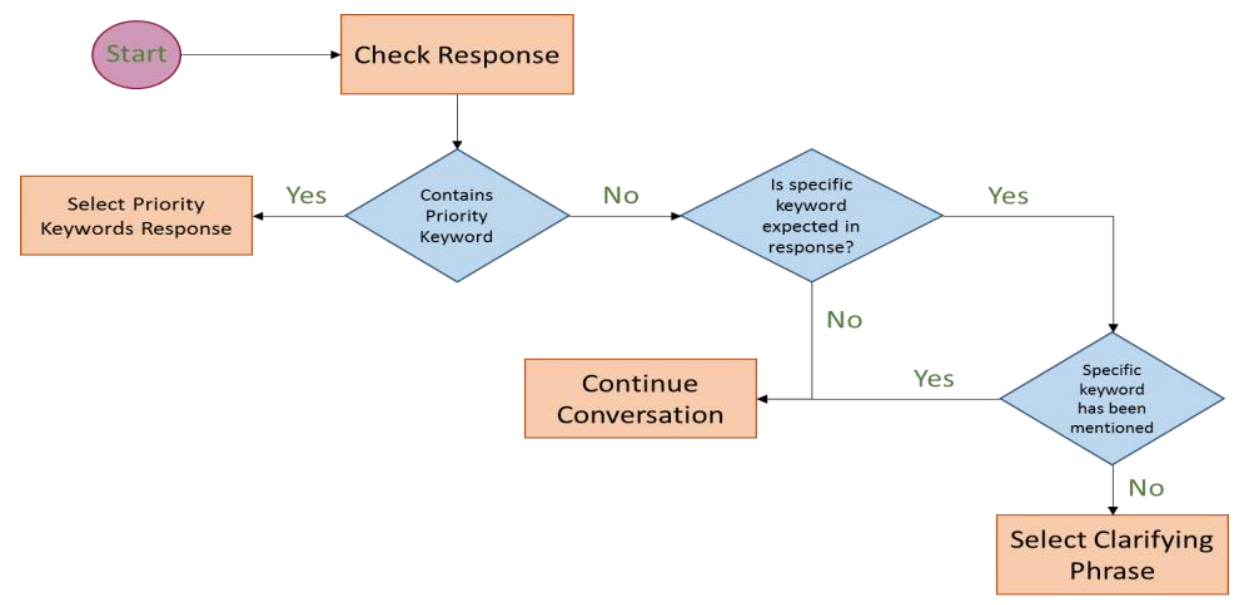

Figure 3. Hello Barbie Keyword Interaction 
to strengthen the connection between Hello Barbie and the user.

After discussing the underlying causes of data privacy and security issues in smart toys, we present a research framework to understand how consumers' perceived risks and benefits of the smart toys may influence their product purchase decision in the next section. We also discuss how some culture difference variables may influence consumers differently in smart toys adoption in different countries.

\section{Research Framework}

Understanding consumers' new product purchase decision is very important for companies to successful design and manages their new products. Previous research shows that consumers' purchase intention towards a new product can be influenced by several factors such as consumers' perceived innovativeness, perceived risk, and benefits of the product $[10,11]$. The degree of the innovativeness of a new product may vary from really new to incrementally new $[19,28]$. Really new products use ground-breaking technologies (e.g., digital camera) or establish new markets with existing technologies (e.g., Sony Walkman) [28]. New products incrementally provide improvements over existing products (i.e., iPhone 7 vs. iPhone 6). Therefore, really new products provide entirely new benefits not available on existing products, whereas incrementally new products enhance the benefits currently offered by existing products. The literature shows that really new products often provides more added benefits to consumers, but at the same time also come with more risks. Ziamou proposes that consumers perceive more benefits from really new products than incrementally new products and thus are more likely to adopt such products [27]. Whereas, other research has demonstrated that consumers may find it difficult to understand the benefits of really new products and are thus more likely to focus on the risks of these products, which then negatively affects consumers' purchase intention toward such products [11].

Hung et al. [29] defined a smart toy as a mobile device consisting of a physical toy component that connects to one or more toy computing services to facilitate gameplay in the Cloud through networking and sensory technologies to enhance the functionality of a traditional toy. They can be considered really new products as smart toys have been categorized as a new market of

\footnotetext{
${ }^{1}$ http://www.toyfairny.com/Toy-

Fair/Home/Toy_Fair/Toy_Fair_2015.aspx

${ }^{2}$ http://www.telegraph.co.uk/technology/google/11633336/Google-has-created-plans-for-a-creepy-toythat-records-your-children.html
}

toys to differentiate from traditional toys. A smart toy can easily capture a child user's physical activity state (e.g., walking, standing, running etc.), store personalized information (e.g., location, activity pattern etc.) through the camera, microphone, Global Positioning System (GPS), and various other sensors. These sensors enable smart toys to monitor and interact with children in ways which were not possible even five years ago. Furthermore, advances in AI functions such as facial and speech recognition enable cloud-based services to integrate this data and have the toy interact 'intelligently' with the user while allowing back-end systems to mine the data for a myriad of other purposes. For example, the Google Toy ${ }^{1}$ has been criticized in the media where people have expressed concerns about Google breaching the expected privacy of such devices [30]. As another example, there is a class action lawsuit alleging that Mattel's Hello Barbie ${ }^{2}$ records children's conversations without parental consent, in violation of the Children's Online Privacy Protection Act (COPPA) in California [31]. Further, Germany's telecommunications watchdog has ordered parents to destroy or disable a "smart doll" because the toy can be used to illegally spy on children ${ }^{3}$.

How innovative consumers perceive smart toys may affect how they evaluate the risks and benefits of smart toys and consequently influencing their purchase intention toward smart toys. As depicted in Figure 4, we designed a research model to examine how consumers' perceived innovativeness of the toy, perceived risks of the conversational function and perception of the conversational function to influence their overall evaluation of the toy, attitudes toward the toy, and purchase intention toward the toy.

Further, the existing literature also recognizes the effect of cultural differences and social contagion on consumer new product adoption process in different countries (e.g., [13]). For example, the uncertainty avoidance dimension of Hofstede's culture typology measures "the extent to which the members of a culture feel threatened by uncertain or unknown situations" $[14,19]$. Therefore, consumers in countries with high uncertainty avoidance scores are less likely to adopt innovations given their risk avoidance nature. As Brazilians score relatively lower than Argentinians on this dimension (76 vs. 86), we argue that Brazilian consumers are more likely to adopt smart toys than Argentinian consumers are, as they are less risk-averse. In addition, the power distance dimension addresses "the extent to which the less

${ }^{3}$ http://www.washingtontimes.com/news/2015/dec/9/hellobarbie-makers-sued-after-security-researcher/ 
powerful members of a culture expect and accept that power is distributed unequally [14]." Research shows
- Perceived risks of the conversational function of the smart toy was measured by three items, "I am

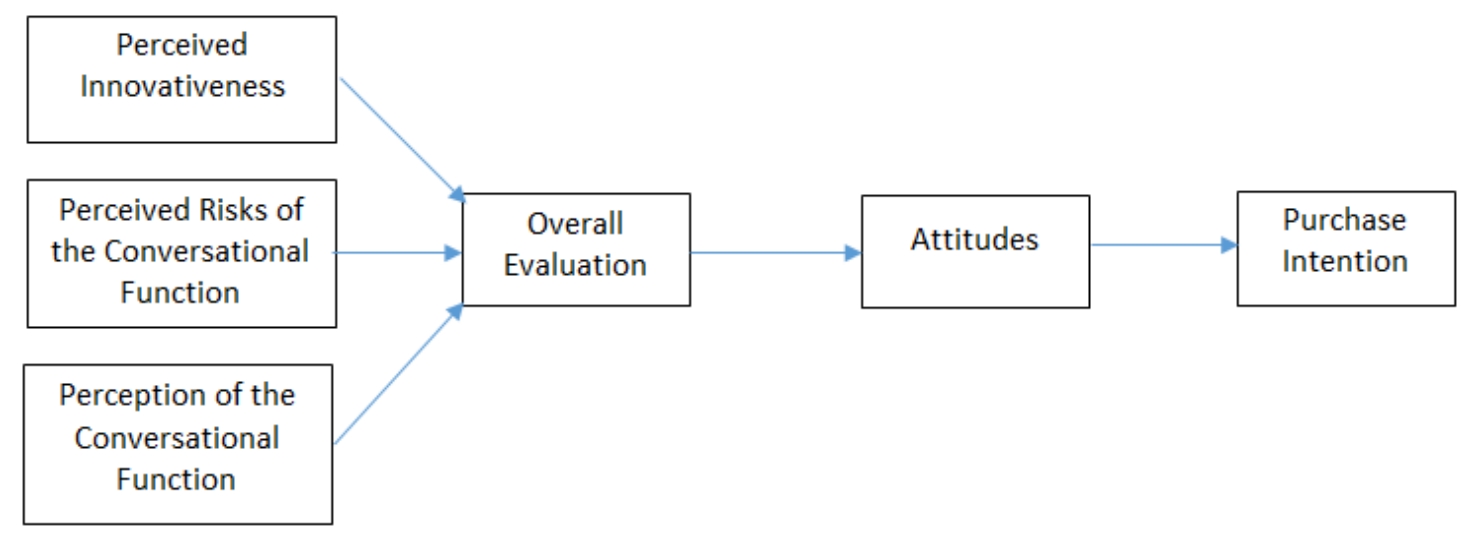

Figure 4. Proposed Research Model

that people in high power distance countries are likely to buy products for its social status and imitate the behavior of other people [13]. As Brazilians score higher than Argentinians on this dimension (69 vs. 49), we expect Brazilian consumers are more likely to adopt smart toys as a status symbol than Argentinian consumers are.

\section{Methods and Results}

We conducted a survey about how consumers perceive Hello Barbie in Brazil and Argentina. We used Hello Barbie as an example of smart toys as smart toys including Hello Barbie have not been widely marketed in these two countries. Thus, we can assess consumers' reaction toward a really new product with networking technologies.

We measured all the variables in our research model (cf. Figure 4) using a 5-point scale with items adapted from a related work [15], which are described as follows:

- Perception of the conversational function of the smart toy was measured by two items, "to what extent does the conversation function of Hello Barbie make sense to you" and "to what extent do you like the conversation function of Hello Barbie" (reliability $=.61)$. A variable, e.g., purchase intention, sometimes is measured by several items (questions). To make sure that these measurement items are consistent with each other, we use reliability as an indicator. Usually, a reliability alpha higher than .7 means reliability, and higher the number the higher the reliability. This number is not used in the analysis, but to show that the measurement items are good. For those variables only measured by one item/question, there is no reliability. afraid/worried that the conversation function including the recording function of Hello Barbie may 'violate the user's personal privacy' / 'gather too much of the user's information' / 'lead to some potential data security issues in the future"' (reliability $=.89$ ).

- Perceived innovativeness of the smart toy was measured by one item, "how innovative do you think Hello Barbie is", ranging from " $1=$ not at all innovative" to " $5=$ very innovative".

- Attitude toward the smart toy was measured by three items asking participants' overall evaluation of the toy being "very bad/very good," "very unfavorable/very favorable," and "not at all appealing/very appealing" (reliability $=.82$ ).

- Overall evaluation of the smart toy considering its benefits and risks was measured by one item, "please provide an overall evaluation of Hello Barbie after considering its benefits and potential risks", ranging from " $1=$ risks outweigh benefits" to " $5=$ benefits outweigh risks".

- Purchase intention toward the smart toy was measured by two items, "how interested will you be in buying a Hello Barbie for yourself or a child" and "what is the probability that you will buy a Hello Barbie for yourself or a child" (reliability = .79).

This study also measured participants' trait innovativeness, history of using smartphone and speech recognition software, whether they had heard of the toy before, and demographic variables as control variables.

We conducted this preliminary study at the University of Sao Paulo in Brazil and National Technological University - Santa Fe in Argentina. Before the survey, we gave a presentation of Hello Barbie's functions and related background information to the participants. Please note that all the participants are the non-English native speaker and they speak either Portuguese or 
Spanish. They are either university faculty members or students. 118 participants $(73.9 \%$ male and $24.6 \%$ female) completed the questionnaire. Among them, 46 (39\%) were from Brazil and $72(61 \%)$ were from Ar- the toy $(3.25$ vs. $2.57, F(1,116)=14.24, p<.001)$ and hence expressed higher purchase intention toward the toy $(2.26$ vs. $1.63, \mathrm{~F}(1,116)=14.17, \mathrm{p}<.001)$ than Argentinian participants did. However, different from our ex-

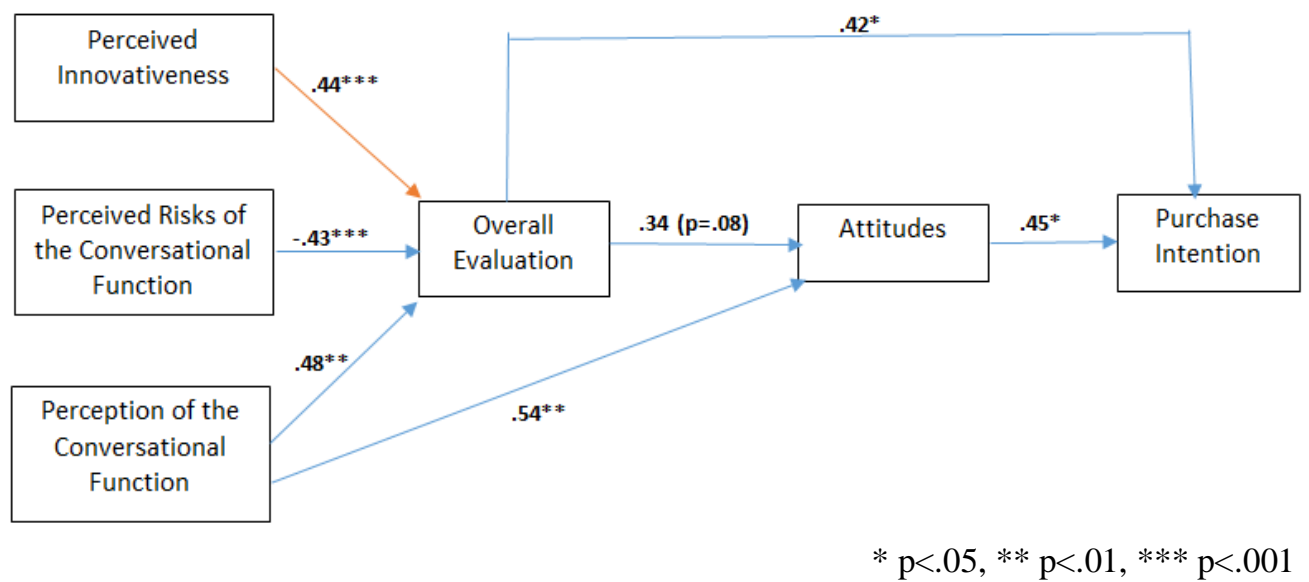

Figure 5. Results in Brazil for structural equation modeling

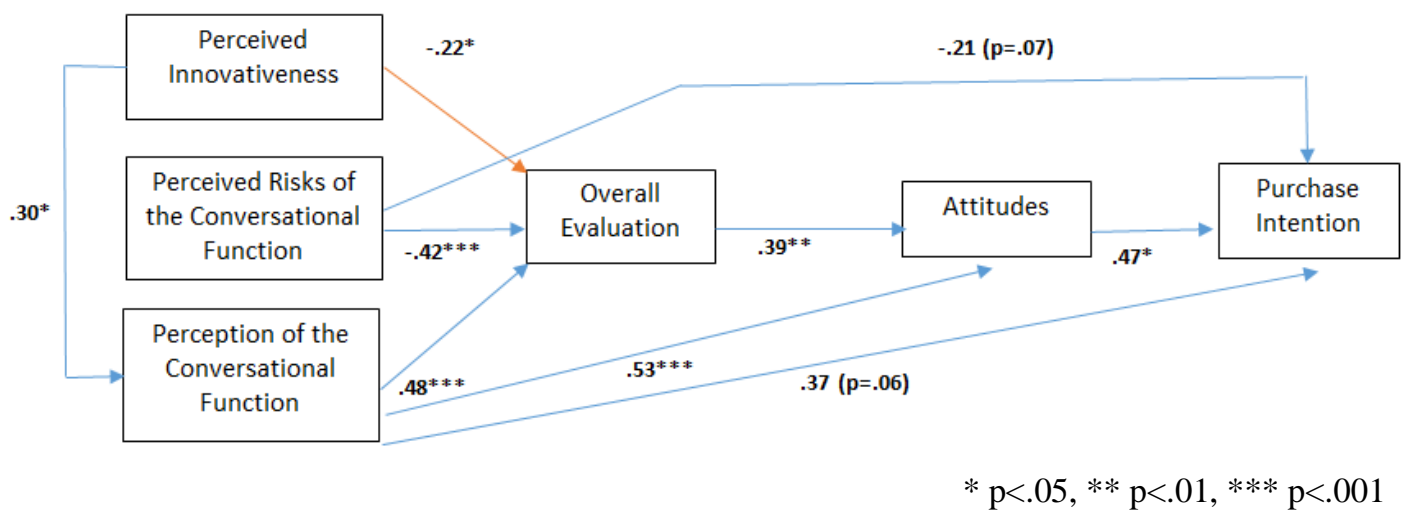

Figure 6. Results in Argentina for structural equation modeling

gentina. The average age was 28 years. We first analyzed whether Brazilian participants perceived Hello Barbie differently from Argentinian participants. Analysis of Covariance (ANCOVA) with perception of the conversational function, perceived risks of the conversational function, perceived innovativeness, overall evaluation, attitudes and purchase intention as separate dependent variables and age, gender, number of children, whether they had seen the toy before, individual trait innovativeness and speech recognition application usage as covariates showed that none of the covariates were significant. Therefore, these covariates were dropped in the analysis and we report the results of analysis of variance (ANOVA). As we have predicted, Brazilian participants perceived the conversational function of the toy better $(3.54$ vs. $2.95, F(1,116)=8.41, \mathrm{p}<.01)$, had better overall evaluation (2.93 vs. 2.15, F(1, $116)=16.25, \mathrm{p}<.001)$, had more positive attitudes toward pectation, there was no significant difference between the Brazilian and Argentinian participants in their perceived risks of the conversational function (4.17 vs. $3.91, \mathrm{~F}(1,116)=1.80, \mathrm{p}>.1)$ and perceived innovativeness of the toy $(3.87$ vs. $4.07, F(1,116)=1.25, \mathrm{p}>.2)$. In other words, participants in both countries assessed the smart toy as equally innovative and risky. Then, why did this equal innovativeness and risk perception lead to different levels of overall evaluation and purchase likelihood? We answer this question in the following analyses.

Second, we tested our proposed research model using structural equation modeling. The results are shown in Figures 5 and 6. All the relationships between variables were tested, but only the significant relationships are presented by lines in the figures. An interesting difference between the two models is that perceived inno- 
vativeness negatively affected people's overall evaluation of the toy in Argentina, but positively affected people's overall evaluation of the toy in Brazil (this relationship is highlighted in orange color in both figures for ease of comparison). This may suggest that although consumers in both countries have similar levels of perceived innovativeness of the toy, Argentinian consumers evaluate the toy worse when they perceive the toy as newer, whereas Brazilian consumers evaluate the toy better when they perceive the toy as newer. In other words, Brazilians like "newer" innovations and such preference contributes to their higher purchase intention. This difference may be explained by the different levels of uncertainty avoidance and power distance between the two countries. As Brazilians score slightly lower on uncertainty avoidance than Argentinians do [14], they may focus more on the benefits side than the risks side when evaluating really new products such as the smart toys. Our results also showed that Brazilians perceived the conversational function better than the Argentinians did. Whereas, Argentinians might have focused more on the risks side when evaluating really new products due to their relatively higher risk avoidance tendency. Further, as discussed earlier Brazilians score relatively higher on the power distance dimension than Argentinians do [14]. Brazilians may be more interested in really new products because the innovativeness nature of really new products can help to serve as a status symbol. Therefore, the more innovative they perceive the smart toys, the better the evaluation and higher purchase intention. Taken together, the culture difference between Brazil and Argentina may help to explain the different consumer reaction towards smart toys in the two countries.

\section{Conclusion and Future Work}

In summary, there are three properties of a smart toy: (1) Pervasive - a smart toy may follow child through everyday activities; (2) Social - social aspects and multiplayer are becoming a mandatory aspect of interactive smart toys in a one-to-one, one-to-many and many-tomany relations [16]; and (3) Connected - Smart toys may connect and communicate with other toys and services through networks. Children provide a unique user base which requires special attention in several key areas related to their privacy. Children's data is widely considered to be particularly sensitive and should be treated with extreme care by law and legislation [17]. Privacy can result in physical safety of child user [18]. A framework is required which can achieve these privacy goals by minimizing the collection and retention of potentially sensitive user data, as well as involving the user (or parent) in the control of their child's data. End- user requirements need to consider that the main user base is children, who have unique requirements as they are especially vulnerable and to protect their sensitive location data, parents/guardians require a method to implement privacy controls on their child's data.

Our empirical study shows that participants in both countries assessed the smart toy as equally innovative and risky. This demonstrates the data privacy concerns in Brazil and Argentina. Further, our research also demonstrates how perceived innovativeness of a product may have either positive or negative impact on product evaluation and purchase intention in diverse cultures.

The results of our empirical study suggest that smart toy manufacturers can emphasize the toy's innovativeness to enhance consumer acceptance level in relatively low uncertainty avoidance cultures and relatively highpower distance countries such as Brazil. Whereas, in cultures with relatively higher uncertainty avoidance and relatively low power distance such as Argentina, smart toy manufacturers can reduce consumers' perceived innovativeness by associating the conversational technology with existing technology such as voice recognition mobile apps to enhance consumers' evaluation of the toy.

By our best knowledge, this is one of the first research attempts to study the perceived innovativeness and privacy risk of smart toys in Brazil and Argentina. There is a limitation in our empirical study. The size of the collected sample data is not large enough to show a full spectrum of results. We will continue to collect sample data from Brazil and Argentina in compliance with the guidelines given by statistical sampling theory as a major future work [32][33].

For other future works, we will collect more data in North America, Asia, and the Middle East to compare the results with South America. Further, we will test different mechanisms (e.g., increasing consumers' perceived control over the data) to determine which one is more effective in mitigating perceived privacy risk in North and South America.

\section{References}

[1] Byrne, C., "Hot toys are dead: long live hot products, Young Consumers," January 2006, vol. 7, no. 1, pp. 8-13

[2] Michael, K. and Hayes, A., "High-Tech Child's Play in the Cloud: Be safe and aware of the difference between virtual and real," IEEE Consumer Electronics Magazine, vol. 5, no. 1, 11 December, 2015, pp. $123-128$

[3] Dudau, V., "German agency tells parents to destroy smart toy due to fears it's being used to spy on kids," Neowin, 2017. Online: https://www.neowin.net/news/german-agency-tellsparents-to-destroy-smart-toy-due-to-fears-its-being-used-tospy-on-kids [Accessed: 18 February, 2017] 
[4] OEC. The Observatory of Economic Complexity, 2017. Online: http://atlas.media.mit.edu/en/ [Accessed: 17 February, 2017]

[5] A\&E Networks. 1959 Barbie makes her debut, 26 November, 2016. Online: Available: http://www.history.com/this-day-in-history/barbie-makes-her-debut [Accessed: 8 February, 2017]

[6] Hains, R., "5 Reasons NOT to Buy Barbie for Little Girls (It's Not Just Body Image!)," 15 December, 2014. Online: Available: https://rebeccahains.com/2014/12/15/5-reasonsnot-to-buy-barbie/ [Accessed: 8 February, 2017]

[7] Mattel. Hello Barbie Messaging Q\&A, December 2015. Online: http://hellobarbiefaq.mattel.com/wp-content/uploads/2015/12/hellobarbie-faq-v3.pdf [Accessed: 8 February, 2017]

[8] CIPP Guideline. Introduction to Privacy Law in Brazil. Online: https://www.cippguide.org/2015/02/23/introductionto-privacy-law-in-brazil/ [Accessed: 8 February, 2017] [9] Goldstein, J., Buckingham, D. and Brougère, G., "Toys, Games, and Media, Lawrence Erlbaum Associates," Publishers, Mahwah, New Jersey London, June 2004, 262 pages [10] Hoeffler, S., "Measuring Preferences for Really New Products," Journal of Marketing Research, Vol. 40, no. 4, 2003, pp. 406-420

[11] Jhang, J. H., Grant, S. J., and Campbell, M. C., "Get It? Got It. Good! Enhancing New Product Acceptance by Facilitating Resolution of Extreme Incongruity," Journal of Marketing Research, Vol. 49, 2012, pp. 247-259

[12] Moreau, C. P., Lehmann, D. R., and Markman, A. B., "Entrenched knowledge structures and consumer response to new products," Journal of Marketing Research, Vol 38, No. 1, 2001, pp. 14-29

[13] Van den Bulte, C. and Stremersch, S., "Social Contagion and Income Heterogeneity in New Product Diffusion: A MetaAnalytic Test," Marketing Science, Vol. 23, no. 4, 2004, pp. 530-544

[14] Hofstede, G., 'Culture's Consequences: Comparing Values, Behaviors, Institutions, and Organizations Across Nations," Second Edition, Thousand Oaks CA: Sage Publications, 2001

[15] Ma, Z., Gill, T. and Jiang, Y. "Core Versus Peripheral Innovations: The Effect of Innovation Locus on Consumer Adoption of New Products," Journal of Marketing Research, vol. 52, no. 3, June 2015, pp. 309-324

[16] Tath, E. I., "Context Data Model for Privacy," PRIME Standardization Workshop, 2006, pp. 1-6

[17] Toy Industry Association, "The Changing Privacy and Data Security Landscape - From Mobile Apps to OBA," Washington, D.C.: Keller and Heckman LLP, 2012. Online: http://www.toyassociation.org/App_Themes/tia/pdfs/priorities/M2C/PrivacyWhitePaper.pdf

[18] McClary, A., "Good Toys, Bad Toys: How Safety, Society, Politics and Fashion Have Reshaped Children's Playthings," McFarland \& Company, May 2004, 201 pages

[19] Hofstede, G., Hofstede, J. H., and Minco, M., "Cultures and Organizations: Software of the Mind. Revised and Expanded," 3rd Edition, McGraw-Hill, 2010

[20] Corbyn, Z., "The Future of Smart Toys and the Battle for Digital Children," The Guardian, Online: https://www.theguardian.com/technology/2016/sep/22/digital-children-smart-toys-technology [Accessed: 8 February, 2017]

[21] eMarketer, “Amazon Echo's Holiday Sales Hint at Huge Market to Come," 2016, Online: https://www.emarketer.com/Article/Amazon-Echos-Holiday-Sales-Hint-HugeMarket-Come/1014957 [Accessed: 8 February, 2017]

[22] Hann, I. H., Hui, K. L., Lee, S. Y. T., and Pang, I. P. L., "Overcoming Online Information Privacy Concerns: An Information-processing Theory Approach," Journal of Management Information Systems, Vol. 24, 2007, pp. 13-42

[23] Juniper Research, "Smart Toy Revenues to Hit \$2.8bn This Year, Driven by Black Friday \& Christmas Holiday Sales," 2017, Online: https://www.juniperresearch.com/press/press-releases/smart-toy-revenues-to-hit\$2-8bn-this-year [Accessed: 8 February, 2017]

[24] Martin, K. D. and Murphy, P.E., "The Role of Data Privacy in Marketing," Journal of the Academy of Marketing Science, Vol. 45, March 2017, pp. 135-155

[25] Nelson, B., "Children's Connected Toys: Data Security and Privacy Concerns," Office of Oversight and Investigations Minority Staff Report, US Senate Committee on Commerce, Science, and Transportation, 2016, Online: https://www.billnelson.senate.gov/sites/default/files/12.14.16_Rank-

ing_Member_Nelson_Report_on_Connected_Toys.pdf[Accessed: 8 February, 2017]

[26] Sheehan, K. and Hoy, M., "Flaming, Complaining, Abstaining: How Online Users Respond to Privacy Concerns," Journal of Advertising, Vol. 28, No. 3, 1999, pp. 37-51

[27] Ziamou, P. L., "The Effect of the Degree of Newness of a 'Really New' Product on Consumers' Judgments," Advances in Consumer Research, Vol. 26, 1999, pp. 368-371

[28] Garcia, R. and Calantone, R., "A Critical Look at Technological Innovation Typology and Innovativeness Terminology: A Literature Review," The Journal of Product Innovation Management, Vol. 19, No. 2, 2002, pp. 110-32

[29] Hung, P. C. K., Fantinato, M., Rafferty, L., Iqbal, F., Kuo, S-Y. and Huang, S-C. (2017). Towards a Privacy Rule Model for Smart Toys. The IEEE 50th Hawaii International Conference on System Sciences (HICSS-50), Big Island, Hawaii, USA, January 4-7, 2017.

[30] Heurix, J., Zimmermann, P., Neubauer, T., and Fenz, S. (2015). A taxonomy for privacy enhancing technologies. Computers and Security, 53, pp. $1-17$.

[31] 12. Smiley, L. (2016). When Toys Talk (And Listen). The California Sunday Magazine, September 29, 2016.

[32] Garvin, J. S., and McClean, S. I., "Convolution and Sampling Theory of the Binormal Distribution as a Prerequisite to Its Application in Statistical Process Control," Journal of the Royal Statistical Society. Series D (The Statistician), Vol. 46, No. 1, 1997, pp. 33-47

[33] Thompson, S. K., "Sampling," Wiley series in probability and statistics, 2nd ed., 2002 